\title{
Branch Atheromatous Disease: A Clinically Meaningful, Yet Unproven Concept
}

\author{
Luca Petrone $^{a}$ Stefania Nannoni ${ }^{a} \quad$ Alessandra Del Bene ${ }^{a}$ Vanessa Palumbo $^{b}$ \\ Domenico Inzitari ${ }^{\text {a }}$ \\ ${ }^{a}$ NEUROFARBA Department, Neuroscience Section, University of Florence, and ${ }^{b}$ Stroke Unit and Neurology, Careggi \\ University Hospital, Florence, Italy
}

\section{Key Words}

Acute ischemic stroke · Advanced MRI · Intracranial atherosclerotic diseases - Vascular risk factors .

Small vessel disease

\begin{abstract}
Background: In 1989, Louis Caplan first used the term branch atheromatous disease (BAD) to describe an occlusion or stenosis at the origin of a deep penetrating artery of the brain, associated with a microatheroma or a junctional plaque, and leading to an internal capsule or pontine small infarct. BAD remained an understudied concept for decades. In recent years, the increasing diffusion of high-resolution magnetic resonance imaging (HRMRI) techniques brought new attention to the BAD debate. We have reviewed clinical studies dealing with BAD-related stroke checking whether a univocal definition of BAD existed, as well as to what extent were consistently associated clinical and imaging features reported. Summary: We conducted a search of the available literature published up to October 20, 2015 via PubMed using the following search terms: 'branch atheromatous disease,' 'intracranial branch atheromatous disease,' 'cerebral branch atheromatous disease,' combined with 'stroke.' Forty-six ar-
\end{abstract}

\section{KARGER}

E-Mail karger@karger.com

www.karger.com/ced ticles were included. We found discrepant definitions and a large variation among clinical features reported in BAD-related stroke patients: among others, a consistent association between BAD and any specific vascular risk factor profile was not detected. Despite this, early neurological deterioration (END) was consistently reported to occur frequently in such patients, although no clear-cut rate range or specific predictor or mechanism of progression was established. In a majority of the studies reporting imaging data, BAD diagnosis was not based on the selective site or type of arterial walls changes, but was inferred based on the vascular territory, size and/ or shape of the ischemic lesion. Following the concept that these changes are seated proximally along the perforator artery, differently from to lipohyalinosis changes located distally, the consequent ischemic lesion was hypothesized to be larger in BAD than in lacunar infarcts. However, across reviewed studies, there was little consistency on the dimensional cutoff used to define BAD-related infarcts. In the last few years, a still limited number of studies using HRMRI techniques is providing preliminary proofs that atheromatous changes causing selective remodeling in the parent vessel and extending through the proximal segment of perforating vessel may subtend BAD. Key Messages: Our literature search showed the lack of a clear-cut definition of BAD, al- 
though BAD-related strokes were consistently considered a high risk of END. The use of high-resolution imaging techniques in the assessment of small subcortical strokes may represent the cornerstone in the perspective to better delimiting the boundaries of BAD as a nosological entity.

(c) 2015 S. Karger AG, Basel

\section{Introduction}

Intracranial branch atheromatous disease (BAD) was first described in 1989 [1] as a mechanism alternative to lipohyalinosis, considered the arteriopathy selectively linked with lacunar infarction pathogenesis. In its initial meaning, the term BAD had been applied to the pathological finding of an occlusion or stenosis at the origin of a deep penetrating artery, due to the presence of a microatheroma or a junctional plaque [2]. Since the first reports by Fisher and Caplan in the 1970s, there has been a distinct lack of further pathological studies on the subject. Thus, BAD remained a neglected concept for decades. The wider diffusion of MRI techniques brought new attention to the BAD debate. The term BAD is being used again to label imaging features supposedly associated with the proximal occlusion of a penetrating artery due to microatheroma or to large parent artery plaque. Routine imaging techniques are unable to depict small vessel changes; thus, features used to define $\mathrm{BAD}$ results to be mostly indirect, consisting of selective morphological characteristics of the ischemic lesion presumed to be produced by it. These characteristics are thought to separate BAD lesions from those related to lipohyalinotic degeneration (true lacunar infarcts). Despite the increasing number of neuroimaging studies, there is still lack of consistency both in the terminology used to describe the aforementioned atheromatous lesions and in the subsequent ischemic infarctions. Very recently, high-resolution (HR) and high-field MRI proved capable of revealing and describing in much more detail intracranial vessels wall changes, including plaques involving perforators' parent arteries [3].

We review and discuss here the definition of $\mathrm{BAD}$, as well as the associated clinical and imaging features.

\section{Materials and Methods}

We conducted a search of the available literature published up to October 20, 2015 via PubMed. We used the following search terms: 'branch atheromatous disease,' 'intracranial branch atheromatous disease,' 'cerebral branch atheromatous disease,' com- bined with 'stroke.' The PubMed search yielded 58 results. We excluded studies according to the following criteria: (1) articles for which an English full translation was not available ( $\mathrm{n}=14)$; (2) studies with no relevance to the review subject, as was evident examining titles and abstracts $(n=8)$. Thus, we identified 36 eligible articles; thorough reading of these articles led to the exclusion of one additional item that did not focus on the topic. Furthermore, 11 articles that were listed in bibliographies of selected works were included because they appeared relevant to the subject (fig. 1).

\section{Clinical Features}

The BAD risk factor profile encompasses common vascular risk factors, such as high blood pressure (HBP), diabetes mellitus (DM), dyslipidemia and tobacco dependence. Among the examined studies, prevalence of HBP ranged from 50 to $92 \%$ in groups of stroke patients whose etiology could be attributed to BAD $[4,5]$. In the same groups, prevalence of DM varied from 23 to $63 \%[6,7]$; smoking habit and dyslipidemia prevalence also varied greatly, ranging from 0 to $50 \%$ and from 17 to $86 \%$ respectively $[8,9]$. Diabetes or an Asian/African ancestry was identified as selectively associated with $\mathrm{BAD}$ since its first description $[1,10]$. More recently, however, a number of studies have reported risk factors similar to those commonly found in both large artery atherosclerosis $[5,11]$ or to lacunar infarctions $[12,13]$. The mean age of onset ranged from 54 to $75[6,13]$; male gender was overall more prevalent. Table 1 summarizes the clinical correlates of $\mathrm{BAD}$, as reported in the examined studies. It is worth noting that all but one of the original studies [9] whose data are shown in table 1 included only patients from Korean, Japanese or Chinese populations.

Early neurological deterioration (END) was reported to occur frequently in BAD-related strokes: the examined studies show an incidence ranging from about 17 to $75 \%$ $[9,14]$. Although mechanisms underlying progression are not completely understood, numerous hypotheses have been formulated, including local thrombosis, local blood-brain barrier disruption, edema, inflammation and excitotoxicity [15]. It has to be noted, however, that the definition of END lacks consistency among different studies: while most of the studies based the definition on a clinical worsening, as measured by the National institute of Health Stroke scale (NIHSS), there is no consensus on the NIHSS cut-off needed to define END in a homogeneous fashion across studies (current definitions range from an increase of at least 1 point in NIHSS motor items to at least 4 points in overall NIHSS score). There is no agreement on the time elapsed from the times of symp-
Petrone/Nannoni/Del Bene/Palumbo/ Inzitari 
Fig. 1. Summary of the literature search.

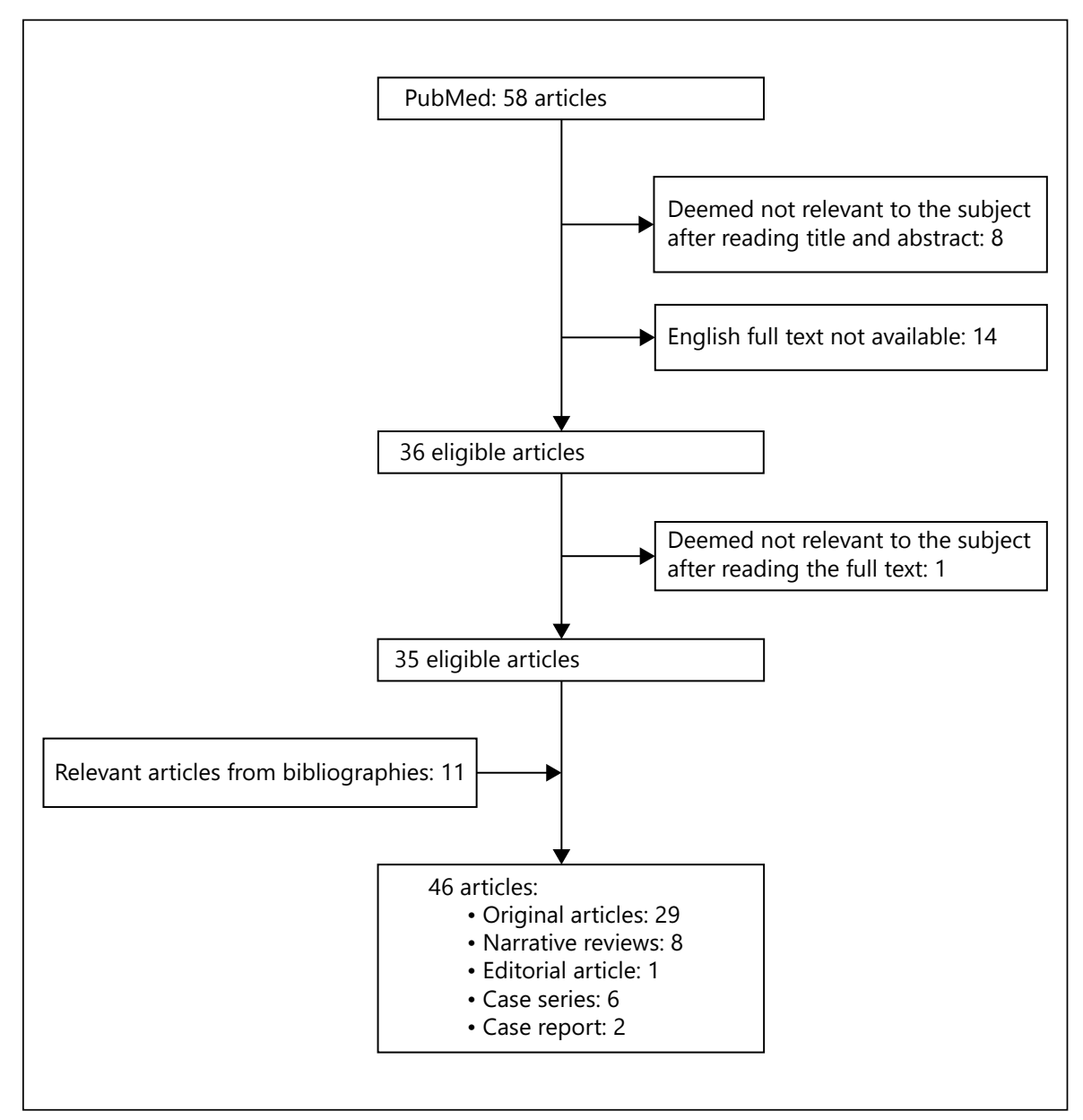

toms onset in relation to which the worsening can be defined 'early' (ranging from an hour to 14 days) $[9,16]$. Thus, comparing studies is difficult and sometimes not viable. From studies that focused on both stroke types, END was definitely more frequent in BAD-related stroke than in true lacunar stroke $[12,13,17,18]$. Yamamoto et al. [19] studied a cohort of 218 patients with what they called large lacunar infarction, identifiable as related to $\mathrm{BAD}$, in order to compare the effect of a combined treatment regimen with cilostazol and edaravone to that of a regimen including either argatroban or ozagrel sodium with regards to the reduction of END. While neither treatment showed a superior effect, in this study, the absence of END was related to a better short-term outcome. Only 2 studies specifically addressed factors potentially involved in END. In a recent study of acute BAD patients [7], an association was reported with an elevated blood homocysteine and a high C-reactive protein level. Nakase et al. [13] studied END in 99 patients with small vessel disease. In the BAD subgroup, mean admission NIHSS score was significantly higher and serum LDL >140 mg/ $\mathrm{dL}$ on admission were significantly more prevalent in patients with END compared with non-deteriorating patients.

Functional prognosis in BAD stroke could be described as halfway between large artery disease-related stroke and lacunar infarctions. Suto et al. [11] conducted a follow-up study of 1,310 stroke patients assessed in the acute phase and followed-up for a mean time of 751 days. Larger subcortical infarction patients (whose etiology can be attributed to BAD) had a short-term prognosis (measured by mRS at discharge) that was similar to that found in atherothrombotic stroke patients, while it was significantly worse than that observed in lacunar infarction patients. On the other hand, in-hospital mortality was similar when compared to large subcortical with lacunar infarctions (0.8 vs. $0 \%)$. Conversely, long-term outcome was reported to be similar to lacunar infarctions (mortal- 
Table 1. Demographic and clinical correlates of BAD

\begin{tabular}{|c|c|c|c|c|c|c|c|c|c|c|}
\hline Kim et al. [6], 1995 & 37 & 13 & 61.5 & 53.9 & 61.5 & 23.1 & 30.8 & n.a. & n.a. & n.a. \\
\hline Lee et al. [40], 2009 & 205 & 22 & n.a. & n.a. & n.a. & n.a. & n.a. & n.a. & n.a. & n.a. \\
\hline Suto et al. [11], 2009 & 1,460 & 138 & 47.8 & 71.0 & 73.9 & 29.7 & 20.2 & 43.8 & 55.4 & Undefined \\
\hline Kim et al. [39], 2009 & 86 & 50 & n.a. & n.a. & n.a. & n.a. & n.a. & n.a. & n.a. & n.a. \\
\hline Aoki et al. [48], 2010 & 51 & 28 & n.a. & n.a. & n.a. & n.a. & n.a. & n.a. & 46.4 & 7 days \\
\hline Kwan et al. [5], 2011 & 372 & 147 & 65.0 & 69 & 92.0 & 36.0 & 43.0 & 67.0 & n.a. & n.a. \\
\hline Yamamoto et al. [49], 2011 & 218 & 218 & 58.2 & 69.2 & 81.5 & 35.7 & 31 & 52.3 & 47.6 & 5 days \\
\hline Deguchi et al. [14], 2013 & 8 & 8 & 63.00 & 70 & 38.0 & 25 & 25 & 50.0 & 75.0 & 7 days \\
\hline Nakase et al. [13], 2013 & 99 & 42 & 59.5 & 73.9 & 76.2 & 42.8 & 45.3 & 42.9 & 38.0 & $48 \mathrm{~h}$ \\
\hline Tanaka et al. [16], 2013 & 242 & 21 & n.a. & n.a. & n.a. & 40.0 & n.a. & n.a. & n.a. & 14 days \\
\hline Miyamoto et al. [45], 2013 & 576 & 32 & n.a. & n.a. & n.a. & n.a. & n.a. & n.a. & 40.6 & 7 days \\
\hline Yoon et al. [50], 2013 & 202 & 28 & 50.5 & 61.7 & 69.2 & 36.3 & 23.1 & 17.6 & n.a. & n.a. \\
\hline Nakase et al. [51], 2014 & 453 & 203 & n.a. & n.a. & n.a. & n.a. & n.a. & n.a. & 67.5 & $48 \mathrm{~h}$ \\
\hline Kim et al. [52], 2013 & 248 & 124 & 46.8 & 65.6 & 60.5 & 23.4 & 25.8 & 39.5 & 16.9 & 5 days \\
\hline Jeong et al. [18], 2015 & 587 & 220 & n.a. & n.a. & n.a. & n.a. & n.a. & n.a. & 21.4 & n.a. \\
\hline Nakase et al. [44], 2015 & 166 & 101 & n.a. & n.a. & n.a. & n.a. & n.a. & n.a. & 45.5 & 7 days \\
\hline Ryoo et al. [42], 2015 & 80 & 36 & 55.6 & 67.6 & 63.9 & 44.4 & 22.2 & 47.2 & n.a. & n.a. \\
\hline Chung et al. [41], 2015 & 286 & 80 & 67.5 & 73.3 & 78.8 & 52.5 & 13.8 & 30 & n.a. & n.a. \\
\hline
\end{tabular}

OTP = Onset to progression; n.a. = non-applicable

ity 8.4 vs. $8.2 \%$; recurrence rate 9.3 vs. $14.1 \%$ ), with significantly lower mortality rate and recurrence rate compared with cardioembolic ( 41.1 and $23.8 \%$ respectively) or atherothrombotic stroke (22.3 and $16.6 \%$ respectively). Likewise, Kwan et al. [5] found that while the length of hospitalization and level of residual disability after rehabilitation were significantly greater in BAD patients than lacunar infarction patients, mortality proved similar between the 2 groups, and lower than mortality observed in large artery stroke patients.

\section{Neuroimaging Features}

Although the term BAD was originally created based on pathological findings, pathological studies have been very few so far. Indeed, our search identified only one such record published in recent years [20]. In contrast, studies based on neuroimaging have been definitely more numerous. In a majority of them, BAD-related infarctions were broadly identified as those occurring in the subcortical regions whose blood perfusion depends on deep small penetrating vessels $[5,21]$. In the anterior circulation, these pertain to lenticulostriate artery terminations stemming from the middle cerebral artery (MCA) and Heubner's artery originating from the anterior cerebral artery [22]. They supply basal ganglia and the internal capsule. In the posterior circulation, thalamoperforating branches, originating from the posterior cerebral artery (thalamic-subthalamic artery), and the posterior communicating artery (tuberothalamic artery) supply the thalamus, while paramedian and short circumferential branches from the basilar artery (BA) supply the pons [23, $24]$. These branches are small enough that an atheromatous lesion of the parent artery involving a branching point can cause ischemia in the whole territory perfused 


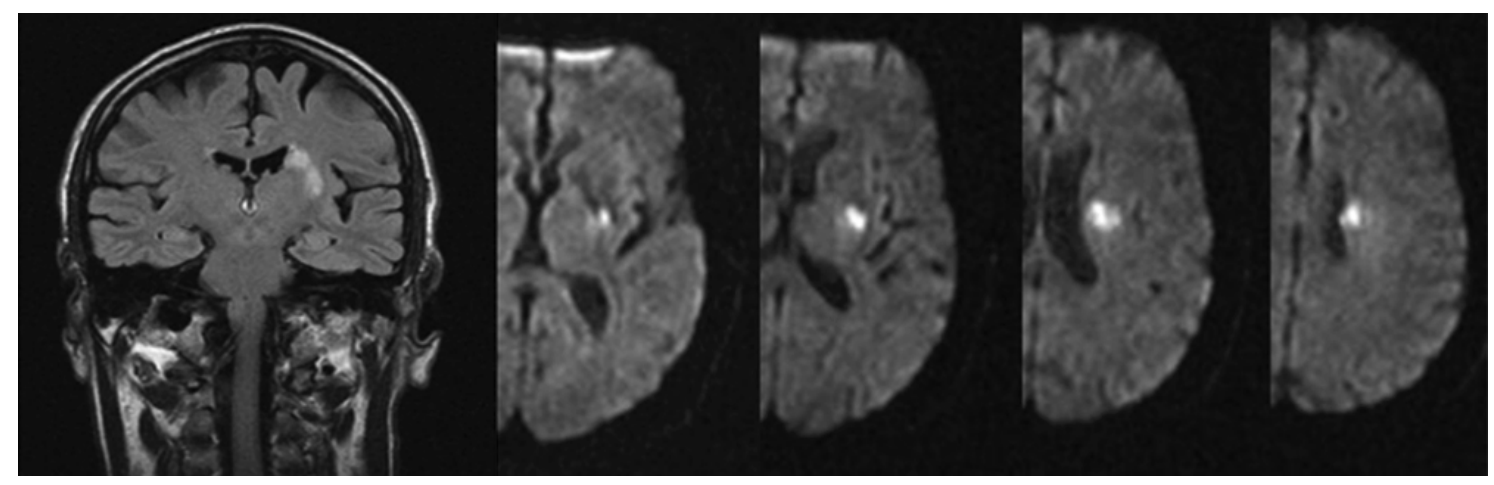

Fig. 2. Example of BAD-related infarction.

by one or few adjacent vessels. Subcortical infarcts can also be caused by occlusion of the anterior choroidal artery (AChA), a larger caliber vessel arising directly from the internal carotid. Specifically, this vessel supplies the posterior part of the corona radiata and of the internal capsule, the tail of the caudate nucleus and the medial part of the globus pallidus, as well as some cortical regions in the temporal cortex [25]. There is no definitive evidence, however, as to what would be the etiology of ischemic events regarding this vessel. In fact, some authors reported that the risk profile and clinical presentation of AChA infarcts are similar to lacunar infarctions [25], while others [26] reported that larger $(>20 \mathrm{~mm})$ AChA lesions may be etiologically linked to large artery embolism or cardioembolism.

$\mathrm{BAD}$ vascular lesions are located proximally along the perforator artery in comparison to lipohyalinosis. Thus, the ensuing ischemic lesion may be expected to be larger in $\mathrm{BAD}$-related infarcts than in lacunar infarcts [27].

There is little consistency on the dimensional cutoff accepted to define BAD-related infarcts. In a majority of the examined works, these lesions were considered identifiable by a diameter $\geq 15 \mathrm{~mm}$ and by the appearance of a lesion in $\geq 3$ consecutive slices on a transversal plane (fig. 2) [28]. It is noteworthy that the thickness of the considered slice is different across the included studies (online suppl. Appendix, see www.karger.com/ doi/10.1159/000442577). Posterior circulation lesions were usually considered to involve unilaterally the pons and to extend to the ventral pontine surface $[12,13,29]$. Table 2 summarizes these data.

Wardlaw et al. [30] recently published the STandards for ReportIng Vascular changes on nEuroimaging (STRIVE) recommendations, in which they proposed an

BAD: A Clinically Meaningful, Yet

Unproven Concept internationally standardized nomenclature for small vessel disease neuroimaging features. Specifically, the term 'small subcortical infarct' is to define small $(<20 \mathrm{~mm}$ transversal diameter) lesions in the territory of a penetrating arteriole. The authors explicitly stated that the proposed definition aims not to imply any specific etiopathogenesis; they also stated that lesions exceeding the $20 \mathrm{~mm}$ limit are to be labeled either striatocapsular infarcts or ACh infarcts according to the appropriate vascular territory. The authors did not suggest a criterion to be applied selectively to BAD-related infarcts.

In fact, currently there is no consensus as to whether BAD should be included either among small vessel or among large vessel intracranial disease [11, 17, 31-34]. Some studies reported BAD as one of the stroke mechanisms linked with intracranial atherosclerotic disease, and characterized by a milder degree of stenosis and comma-shaped infarcts extending to the basal surface of the parent artery [34, 35]. Considering common imaging markers of small vessel disease, Nah et al. [27] retrospectively reviewed data from 805 small subcortical stroke patients, 114 of whom were attributed to BAD. Small vessel disease features such as leukoaraiosis and microbleeds were significantly less prevalent among $\mathrm{BAD}$ patients, while these patients showed a significantly larger atherosclerotic burden, that is a higher prevalence of asymptomatic intracranial atherosclerosis or coronary artery disease. In contrast, Men et al. [29] observed an unusually high prevalence of leukoaraiosis (86.4\%) in a retrospectively analyzed series of 220 BAD Chinese patients. These striking discrepancies likely reflect different inclusion criteria: in fact, some of the studies we examined excluded the diagnosis of BAD on the basis of laboratory data suggestive for widespread large artery disease (table 2).

Cerebrovasc Dis 2016;41:87-95 DOI: $10.1159 / 000442577$ 
Table 2. Neuroimaging features used to define BAD-related infarctions across studies

\begin{tabular}{|c|c|c|c|c|c|c|c|}
\hline \multirow{2}{*}{$\begin{array}{l}\text { First author, } \\
\text { year }\end{array}$} & \multicolumn{5}{|c|}{ Lesion location and size } & \multirow{2}{*}{$\begin{array}{l}\text { Large } \\
\text { vessel } \\
\text { disease } \\
\text { excluded }\end{array}$} & \multirow[t]{2}{*}{ HR MRI } \\
\hline & vascular territory & $\begin{array}{l}\text { transversal } \\
\text { diameter, } \mathrm{mm}\end{array}$ & $\begin{array}{l}\text { longitudinal } \\
\text { diameter }\end{array}$ & $\begin{array}{l}\text { vascular } \\
\text { territory }\end{array}$ & $\begin{array}{l}\text { extension to } \\
\text { ventral surface }\end{array}$ & & \\
\hline Kim et al. [6], 1995 & - & - & - & VB perforators & Yes & NS & No \\
\hline Lee et al. [40], 2009 & - & - & - & PCA perforators & NS & No & Yes \\
\hline Suto et al. [11], 2009 & MCA perforators & $\geq 15$ & NS & VB perforators & NS & Yes & No \\
\hline Aoki et al. [48], 2010 & - & - & - & VB perforators & Yes & No & No \\
\hline Yamamoto et al. [12], 2011 & MCA perforators & $>10$ and $\leq 20$ & $\geq 3$ slices & VB perforators & Yes & Yes & No \\
\hline Chung et al. [8], 2012 & MCA perforators & $\leq 20$ & NS & VB perforators & Yes & Yes & Yes \\
\hline Oyama et al. [33], 2013 & MCA perforators & NS & $>20 \mathrm{~mm}$ & - & - & No & No \\
\hline Deguchi et al. [14], 2013 & MCA perforators & NS & $\geq 3$ slices & VB perforators & Yes & Yes & No \\
\hline Men et al. [29], 2013 & MCA perforators & $\geq 15$ & $\geq 3$ slices & VB perforators & Yes & Yes & No \\
\hline Nakase et al. [13], 2013 & MCA perforators & $\geq 15$ & $>3$ slices & VB perforators & Yes & Yes & No \\
\hline Tanaka et al. [16], 2013 & MCA perforators & $>10$ & $\geq 3$ slices & VB perforators & Yes & Yes & No \\
\hline Yoon et al. [50], 2013 & MCA perforators & NS & $\geq 4$ slices & VB perforators & Yes & Yes & No \\
\hline Chung et al. [41], 2015 & - & - & - & VB perforators & Yes & Yes & Yes \\
\hline
\end{tabular}

HA = Heubner's artery; VB = vertebro-basilar; PCA = posterior cerebral artery; NS = non-specified; n.a. = non-applicable.

Current imaging techniques such as CT angiography, digital subtraction angiography and magnetic resonance angiography (MRA) are of limited utility in the diagnosis of the arteriopathy supposed to subside BAD, because they depict just the external morphology of arteries, and are unable to show inner vessel wall changes. Recently, 3-tesla HR MRI has been used for the visualization of inner MCA and BA plaques in vivo. Klein et al. [3] were able to image MCA atheromatous plaques using HR MRI in 6 patients with MCA stenosis documented by MRA and transcranial doppler. Klein et al. [36] were also able to visualize inner atherosclerotic lesions in the basilar arteries of patients with paramedian pontine infarctions whose MRA had showed normal vessel's profile. They found that either a BA plaque or a wall contrast enhancement was visible on 13 such patients out of a sample of 17 . More recently, Chung et al. [8] performed 3 Tesla HR MRI scans on 15 patients with acute small subcortical infarctions and no relevant vessel disease on MRA (MCA or BA). They identified a relevant branch atheromatous 


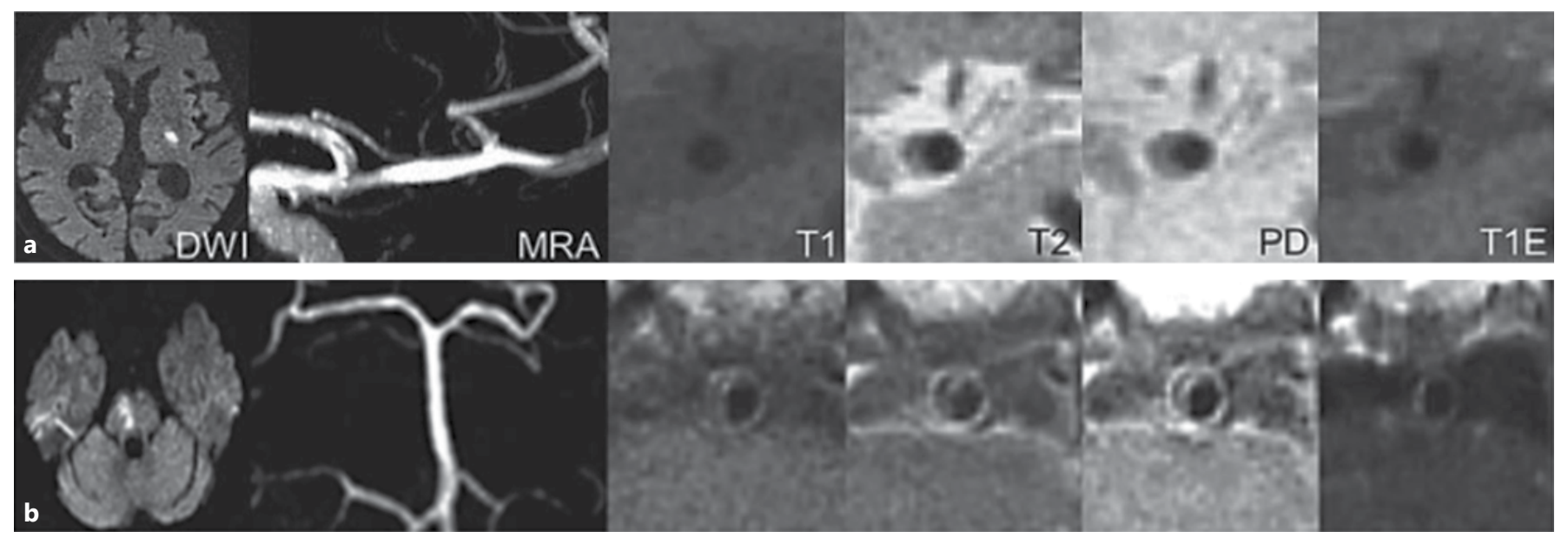

Fig. 3. HR MRI findings of BAD-related infarct and branch atheromatous plaque in the middle cerebral artery (a) and in the basilar artery (b).

plaque in 9 subjects (fig. 3). Niizuma et al. [37] obtained similar results from a small case series: the use of 3 Tesla HR MRI was able to detect MCA plaques in 2 of 3 patients with acute MCA perforator territory infarcts; in one of them, no significant stenosis of the intracranial arteries was apparent on MRA. Similarly, Miyaji et al. [38] reported a patient with acute paramedian pontine infarction in whom HR MRI revealed the presence of BA plaque spreading to the origin of a paramedian pontine artery. We found 3 articles examining clinical and neuroimaging features of different types of vertebro-basilar strokes. In the studies by Kim et al. [39] and by Lee et al. [40], the diagnosis of $\mathrm{BAD}$ was assumed in the presence of an infarction in the territory of one or a few perforating branches arising from a stenosed (of any degree) major vessel (vertebral, basilar or posterior cerebral artery). Chung et al. [41] included patients with posterior circulation stroke and identified BAD by the presence of wall thickening of the BA at the level of the infarct. All these studies used both 1.5 and 3 Tesla MRI although the number of patients studied using HR MRI was not specified. A recent study using HR imaging in 36 patients with deep infarctions within the striatocapsular territory has reported about the presence of the negative type of arterial remodeling (i.e. just limited to the inner arterial wall, and not producing modification of the external vessel profile) in the parent artery [42]. This could be the selective change involved in $\mathrm{BAD}$, and may explain why the vessel caliber may appear normal using current MRA techniques.

As far as END is concerned, we found almost no or very little evidence regarding morphological imaging fea- tures predicting END in BAD-related infarcts. An acute increase in lesion size has been reported as the morphologic hallmark of END in ischemic stroke [43, 44]; Yamamoto et al. [12] observed an association between END and radiologically defined BAD. In a series of 587 with small subcortical infarction, Jeong et al. [18] identified the presence of $\mathrm{BAD}$ or relevant artery stenosis as predictor of END. Miyamoto et al. [45] studied 536 stroke patients looking for clinical and radiological predictors. They also observed a significantly higher prevalence of $\mathrm{BAD}$ among patients whose condition deteriorated. They found a significant association between neurological deterioration and a lesion diameter between 15 and $30 \mathrm{~mm}$. Recently, Feng et al. [46] identified leukoaraiosis severity as a predictor of neurological deterioration in small $(<15$ $\mathrm{mm}$ ) subcortical infarctions. To the best of our knowledge, no study has hitherto specifically investigated early clinical or imaging predictors of END in BAD patients.

\section{Discussion and Conclusions}

Based on our literature search, we observed a large variability of the clinical features in patients where strokes were attributed to BAD. In particular, we were unable to identify a consistent association between BAD and any specific vascular risk factor profile. It is therefore difficult to discuss BAD epidemiology and related clinical correlates.

Although no specific predictor or mechanism of progression has been identified, we can conclude that END 
is importantly frequent in BAD-related strokes. This seemed the most consistent data emerging from our review, definition discrepancies notwithstanding.

Among heterogeneities that emerge from our review, we have to point out that an agreement does not exist either as to whether BAD is an aspect of large or small vessels disease.

While BAD radiological diagnosis was based until now on vascular territory, dimensions and/or shape of the acute ischemic lesion, since conventional imaging techniques can only show indirect evidence of parent artery disease [12, 31, 47], there is increasing evidence that high field and HR MRI techniques may allow direct exploration of at least the atheromatous changes inner to parent vessel that are supposed to subtend BAD [34, 38]. Finally, although most authors identify dimensional limits to describe BAD-related lesions, there is no consensus on the exact values of such limits.

It is not possible to perform a comparison between studies where BAD is confirmed by arteries' inner wall imaging with those where $\mathrm{BAD}$ was only suspected based on the features of the subcortical infarction due to the large inhomogeneity of collected data, as shown in tables 1 and 2. Out of 6 articles using HR MRI to study $\mathrm{BAD}$, only 3 reported clinical features $[8,41,42]$. In the small series studied by Chung et al. [8], where 2 groups of patients with and without HR MRI proved BAD were compared, no patient had potential sources of heart embolism, while other major risk factors such as hypertension and diabetes were equally distributed. Similarly, Ryoo et al. [42] found no significant differences about vascular risk factors distribution between 36 patients with confirmed BAD and 44 patients with non-BAD intracranial atherosclerotic disease. Chung et al. [41] studied 286 patients with posterior circulation stroke of whom 80 with BA atheromatous branch occlusive disease. The comparison of clinical characteristics showed no difference in the distribution of vascular risk factors and stroke severity among the different etiology groups examined.

Despite the almost general assumption that END is particularly frequent in BAD-related stroke, no firm data are available from reviewed studies about its rate range.

Considering the whole material examined in our review, we are confident that HR MRI may represent the switchover leading eventually to a clear and reproducible definition of BAD that might match the original description done in pathology studies. This will hopefully help in conclusively identifying BAD-related strokes, discriminating it reliably from lacunar infarctions due to lipohyalinosis, or from other types of subcortical strokes.

As a translational further objective emerging from our review, we can suggest an ad hoc prospective study enrolling an adequately large sample of patients presenting with small subcortical infarct studied, and using HR MRI combined with clinical data collection. This study should describe carefully the clinical features, risk factors and stroke mechanisms associated with it, and follow up patients for an appropriately long duration to estimate the selective outcomes.

\section{References}

1 Caplan LR: Intracranial branch atheromatous disease: a neglected, understudied, and underused concept. Neurology 1989;39:12461250 .

2 Fisher CM, Caplan LR: Basilar artery branch occlusion: a cause of pontine infarction. Neurology 1971;21:900-905.

3 Klein IF, Lavallée PC, Touboul PJ, Schouman-Claeys E, Amarenco P: In vivo middle cerebral artery plaque imaging by high-resolution MRI. Neurology 2006;67:327-329.

4 Nakase T, Yamazaki T, Ogura N, Suzuki A, Nagata K: The impact of inflammation on the pathogenesis and prognosis of ischemic stroke. J Neurol Sci 2008;271:104-109.

5 Kwan MW, Mak W, Cheung RT, Ho SL: Ischemic stroke related to intracranial branch atheromatous disease and comparison with large and small artery diseases. J Neurol Sci 2011; 303:80-84.
6 Kim JS, Lee JH, Im JH, Lee MC: Syndromes of pontine base infarction. A clinical-radiological correlation study. Stroke 1995;26:950955.

7 Men X, Li J, Zhang B, Zhang L, Li H, Lu Z: Homocysteine and $\mathrm{C}$-reactive protein associated with progression and prognosis of intracranial branch atheromatous disease. PLoS One 2013;8:e73030.

8 Chung JW, Kim BJ, Sohn CH, Yoon BW, Lee SH: Branch atheromatous plaque: a major cause of lacunar infarction (high-resolution MRI study). Cerebrovasc Dis Extra 2012;2: 36-44.

9 Kumral E, Afsar N, Kirbas D, Balkir K, Ozdemirkiran T: Spectrum of medial medullary infarction: clinical and magnetic resonance imaging findings. J Neurol 2002;249:85-93.

10 Caplan LR: Diabetes and brain ischemia. Diabetes 1996;45(suppl 3):S95-S97.
11 Suto Y, Nakayasu H, Maeda M, Kusumi M, Kowa H, Awaki E, Saito J, Nakashima K: Long-term prognosis of patients with large subcortical infarctions. Eur Neurol 2009;62: 304-310.

12 Yamamoto Y, Ohara T, Hamanaka M, Hosomi A, Tamura A, Akiguchi I: Characteristics of intracranial branch atheromatous disease and its association with progressive motor deficits. J Neurol Sci 2011;304:78-82.

13 Nakase T, Yoshioka S, Sasaki M, Suzuki A: Clinical evaluation of lacunar infarction and branch atheromatous disease. J Stroke Cerebrovasc Dis 2013;22:406-412.

14 Deguchi I, Hayashi T, Kato Y, Nagoya H, Ohe Y, Fukuoka T, Maruyama H, Horiuchi Y, Tanahashi N: Treatment outcomes of tissue plasminogen activator infusion for branch atheromatous disease. J Stroke Cerebrovasc Dis 2013;22:e168-e172. 
15 Del Bene A, Palumbo V, Lamassa M, Saia V, Piccardi B, Inzitari D: Progressive lacunar stroke: review of mechanisms, prognostic features, and putative treatments. Int J Stroke 2012;7:321-329.

16 Tanaka R, Ueno Y, Miyamoto N, Yamashiro K, Tanaka Y, Shimura H, Hattori N, Urabe T: Impact of diabetes and prediabetes on the short-term prognosis in patients with acute ischemic stroke. J Neurol Sci 2013;332:45-50.

17 Kim JS, Yoon Y: Single subcortical infarction associated with parental arterial disease: important yet neglected sub-type of atherothrombotic stroke. Int J Stroke 2013;8:197203.

18 Jeong HG, Kim BJ, Yang MH, Han MK, Bae HJ: Neuroimaging markers for early neurologic deterioration in single small subcortical infarction. Stroke 2015;46:687-691.

19 Yamamoto Y, Nagakane Y, Makino M, Ohara T, Koizumi T, Makita N, Akiguchi I: Aggressive antiplatelet treatment for acute branch atheromatous disease type infarcts: a 12-year prospective study. Int J Stroke 2014;9:E8.

20 Tatsumi S, Yamamoto T: An autopsied case of an apparent pontine branch atheromatous disease. Eur Neurol 2010;63:184-185.

21 Arboix A, Blanco-Rojas L, Martí-Vilalta JL: Advancements in understanding the mechanisms of symptomatic lacunar ischemic stroke: translation of knowledge to prevention strategies. Expert Rev Neurother 2014; 14:261-276.

22 Cohen JE, Rabinstein A, Gomori JM, Leker RR: Capsular warning syndrome and crescendo lacunar strokes after atherosclerotic stenosis of the recurrent artery of Heubner. J Clin Neurosci 2012;19:1730-1733.

23 Gállego Culleré J, Erro Aguirre ME: Basilar branch occlusion. Curr Treat Options Cardiovasc Med 2011;13:247-260.

24 Tatu L, Moulin T, Bogousslavsky J, Duvernoy $\mathrm{H}$ : Arterial territories of the human brain: cerebral hemispheres. Neurology 1998;50: 1699-1708.

25 Chausson N, Joux J, Saint-Vil M, Edimonana M, Jeannin S, Aveillan M, Cabre P, Olindo S, Smadja D: Infarction in the anterior choroidal artery territory: clinical progression and prognosis factors. J Stroke Cerebrovasc Dis 2014;23:2012-2017.

26 Ois A, Cuadrado-Godia E, Solano A, PerichAlsina X, Roquer J: Acute ischemic stroke in anterior choroidal artery territory. J Neurol Sci 2009;281:80-84.

27 Nah HW, Kang DW, Kwon SU, Kim JS: Diversity of single small subcortical infarctions according to infarct location and parent artery disease: analysis of indicators for small vessel disease and atherosclerosis. Stroke 2010;41:2822-2827.
28 Nannoni S, Del Bene A, Palumbo V, Petrone L, Sottile F, Pracucci G, Inzitari D: Predictors of progression in patients presenting with minor subcortical stroke. Acta Neurol Scand 2015;132:304-309.

29 Men X, Wu A, Zhang B, Li H, Zhang L, Chen S, Lin Y, Lu Z: Leukoaraiosis and NIHSS score help to differentiate subtypes of intracranial branch atheromatous disease in Southern Han Chinese patients with stroke. Neurol Sci 2013;34:1727-1733.

30 Wardlaw JM, Smith EE, Biessels GJ, Cordonnier C, et al; Standards for ReportIng Vascular changes on Neuroimaging (STRIVE v1): Neuroimaging standards for research into small vessel disease and its contribution to ageing and neurodegeneration. Lancet Neurol 2013;12:822-838.

31 Kim BJ, Kim JS: Ischemic stroke subtype classification: an Asian viewpoint. J Stroke 2014; 16:8-17.

32 Ogata J, Yamanishi H, Ishibashi-Ueda H: Review: role of cerebral vessels in ischaemic injury of the brain. Neuropathol Appl Neurobiol 2011;37:40-55.

33 Oyama H, Kito A, Maki H, Hattori K, Noda T, Wada K: Atherothrombotic lesion of the middle cerebral artery: report of 21 cases with stenotic and obstructive lesions. Nagoya J Med Sci 2013;75:41-46.

34 Bang OY: Intracranial atherosclerosis: current understanding and perspectives. J Stroke 2014;16:27-35.

35 Tamura A, Yamamoto Y, Nagakane Y, Takezawa H, Koizumi T, Makita N, Makino M: The relationship between neurological worsening and lesion patterns in patients with acute middle cerebral artery stenosis. Cerebrovasc Dis 2013;35:268-275.

36 Klein IF, Lavallée PC, Mazighi M, SchoumanClaeys E, Labreuche J, Amarenco P: Basilar artery atherosclerotic plaques in paramedian and lacunar pontine infarctions: a high-resolution MRI study. Stroke 2010;41:1405-1409.

37 Niizuma K, Shimizu H, Takada S, Tominaga T: Middle cerebral artery plaque imaging using 3-Tesla high-resolution MRI. J Clin Neurosci 2008;15:1137-1141.

38 Miyaji Y, Kawabata Y, Joki H, Seki S, Mori K, Kamide T, Tamase A, Nomura M, Kitamura Y, Tanaka F: High-resolution magnetic resonance imaging findings of basilar artery plaque in a patient with branch atheromatous disease: a case report. J Med Case Rep 2014; 29:395.

39 Kim JS, Han YS: Medial medullary infarction: clinical, imaging, and outcome study in 86 consecutive patients. Stroke 2009;40:32213225.

40 Lee E, Kang DW, Kwon SU, Kim JS: Posterior cerebral artery infarction: diffusion-weighted MRI analysis of 205 patients. Cerebrovasc Dis 2009;28:298-305.
41 Chung CP, Yong CS, Chang FC, Sheng WY, Huang HC, Tsai JY, Hsu HY, Hu HH: Stroke etiology is associated with outcome in posterior circulation stroke. Ann Clin Transl Neurol 2015;2:510-517.

42 Ryoo S, Lee MJ, Cha J, Jeon P, Bang OY: Differential vascular pathophysiologic types of intracranial atherosclerotic stroke: a high-resolution wall magnetic resonance imaging study. Stroke 2015;46:2815-2821.

43 Saia V, Pantoni L: Progressive stroke in pontine infarction. Acta Neurol Scand 2009;120: 213-215.

44 Nakase T, Yamamoto Y, Takagi M; Japan Branch Atheromatous Disease Registry Collaborators: The impact of diagnosing branch atheromatous disease for predicting prognosis. J Stroke Cerebrovasc Dis 2015;24:24232428.

45 Miyamoto N, Tanaka Y, Ueno Y, Kawamura M, Shimada Y, Tanaka R, Hattori N, Urabe T: Demographic, clinical, and radiologic predictors of neurologic deterioration in patients with acute ischemic stroke. J Stroke Cerebrovasc Dis 2013;22:205-210.

46 Feng C, Tan Y, Wu YF, Xu Y, Hua T, Huang J, Liu XY: Leukoaraiosis correlates with the neurologic deterioration after small subcortical infarction. J Stroke Cerebrovasc Dis 2014; 23:1513-1518.

47 Kato H, Takeda T, Ohara K, Tei H, Nishizawa E: Rostrocaudal thickness on sagittal diffusion-weighted imaging as a predictor of motor deficits in an acute isolated pontine infarction. J Stroke Cerebrovasc Dis 2015;24:622628.

48 Aoki J, Iguchi Y, Kimura K, Shibazaki K, Iwanaga T, Sakai K: Diameter of the basilar artery may be associated with neurological deterioration in acute pontine infarction. Eur Neurol 2010;63:221-226.

49 Yamamoto Y, Ohara T, Ishii R, Tanaka E, Murai T, Morii F, Tamura A, Oohara R: A combined treatment for acute larger lacunartype infarction. J Stroke Cerebrovasc Dis 2011;20:387-394.

50 Yoon Y, Lee DH, Kang DW, Kwon SU, Suh DC, Bang OY, Kim JS: Stroke recurrence patterns are predicted by the subtypes and mechanisms of the past, non-cardiogenic stroke. Eur J Neurol 2013;20:928-934.

51 Nakase T, Sasaki M, Suzuki A: The effect of acute medication with cilostazol, an antiplatelet drug, on the outcome of small vessel brain infarction. J Stroke Cerebrovasc Dis 2014;23:1409-1415.

52 Kim DE, Choi MJ, Kim JT, Chang J, Park MS Choi KH, Oh DS, Lee SH, Cho KH: Two different clinical entities of small vessel occlusion in TOAST classification. Clin Neurol Neurosurg 2013;115:1686-1692. 